\title{
Commutator Subgroups of Generalized Hecke and Extended Generalized Hecke Groups
}

\author{
Şule Kaymak (Sarıca), Bilal Demir, Özden Koruoğlu and \\ Recep Şahin
}

\begin{abstract}
Let $p$ and $q$ be integers such that $2 \leq p \leq q, p+q>4$ and let $H_{p, q}$ be the generalized Hecke group associated to $p$ and $q$. The generalized Hecke group $H_{p, q}$ is generated by $X(z)=-\left(z-\lambda_{p}\right)^{-1}$ and $Y(z)=-(z+$ $\left.\lambda_{q}\right)^{-1}$ where $\lambda_{p}=2 \cos \frac{\pi}{p}$ and $\lambda_{q}=2 \cos \frac{\pi}{q}$. The extended generalized Hecke group $\bar{H}_{p, q}$ is obtained by adding the reflection $R(z)=1 / \bar{z}$ to the generators of generalized Hecke group $H_{p, q}$. In this paper, we study the commutator subgroups of generalized Hecke groups $H_{p, q}$ and extended generalized Hecke groups $\bar{H}_{p, q}$.
\end{abstract}

\section{Introduction}

In [5], Hecke introduced the groups $H(\lambda)$ generated by two linear fractional transformations

$$
T(z)=-\frac{1}{z} \text { and } U(z)=z+\lambda
$$

where $\lambda$ is a fixed positive real number. Let $S=T U$, i.e.,

$$
S(z)=-\frac{1}{z+\lambda} .
$$

Key Words: Generalized Hecke groups, Extended generalized Hecke groups, commutator subgroups.

2010 Mathematics Subject Classification: 20H10, 11F06, 30F35

Received: 06.02.2016

Revised: 15.06 .2017

Accepted: 20.06.2017 
Hecke showed that $H(\lambda)$ is discrete if and only if $\lambda=\lambda_{q}=2 \cos \left(\frac{\pi}{q}\right), q \geq 3$ integer, or $\lambda \geq 2$. We consider the former case $q \geq 3$ integer and we denote it by $H_{q}=H\left(\lambda_{q}\right)$. The Hecke group $H_{q}$ is isomorphic to the free product of two finite cyclic groups of orders 2 and $q$,

$$
H_{q}=<T, S: T^{2}=S^{q}=I>\simeq C_{2} * C_{q} .
$$

The first few Hecke groups $H_{q}$ are $H_{3}=\Gamma=P S L(2, \mathbb{Z})$ (the modular group), $H_{4}=H(\sqrt{2}), H_{5}=H\left(\frac{1+\sqrt{5}}{2}\right)$, and $H_{6}=H(\sqrt{3})$. It is clear from the above that $H_{q} \subset P S L\left(2, \mathbb{Z}\left[\lambda_{q}\right]\right)$ unlike in the modular group case (the case $q=3)$, the inclusion is strict and the index $\left|P S L\left(2, \mathbb{Z}\left[\lambda_{q}\right]\right): H_{q}\right|$ is infinite as $H_{q}$ is discrete whereas $P S L\left(2, \mathbb{Z}\left[\lambda_{q}\right]\right)$ is not for $q \geq 4$.

Lehner studied in [11] a more general class $H_{p, q}$ of Hecke groups $H_{q}$, by taking

$$
X=\frac{-1}{z-\lambda_{p}} \text { and } V=z+\lambda_{p}+\lambda_{q},
$$

where $2 \leq p \leq q, p+q>4$. Here if we take $Y=X V=-\frac{1}{z+\lambda_{q}}$, then we have the presentation,

$$
H_{p, q}=<X, Y: X^{p}=Y^{q}=I>\simeq C_{p} * C_{q} .
$$

In particular $H_{p, q}$ has the signature $(0 ; p, q, \infty)$. We call these groups as generalized Hecke groups $H_{p, q}$. We know from [11] that $H_{2, q}=H_{q}$, $\left|H_{q}: H_{q, q}\right|=2$, and there is no group $H_{2,2}$. Furthermore all Hecke groups $H_{q}$ are included in generalized Hecke groups $H_{p, q}$. Generalized Hecke groups $H_{p, q}$ have been also studied by Calta and Schmidt in [2] and [3].

Extended generalized Hecke groups $\bar{H}_{p, q}$ can be defined similar to extended Hecke groups $\bar{H}_{q}$, by adding the reflection $R(z)=1 / \bar{z}$ to the generators of generalized Hecke group $H_{p, q}$. Hence extended generalized Hecke groups $\bar{H}_{p, q}$ have a presentation

$$
\bar{H}_{p, q}=<X, Y, R: X^{p}=Y^{q}=R^{2}=I, R X=X^{-1} R, R Y=Y^{-1} R>,
$$

that is

$$
\bar{H}_{p, q}=<X, Y, R: X^{p}=Y^{q}=R^{2}=(X R)^{2}=(Y R)^{2}=I>\cong D_{p} *_{\mathbb{Z}_{2}} D_{q} .
$$

The groups $H_{p, q}$ is a subgroup of index 2 in $\bar{H}_{p, q}$.

Now we focus on the commutator subgroups of $H_{p, q}$ and $\bar{H}_{p, q}$. The commutator subgroups of $H_{q}, \bar{H}_{q}, H_{q}^{m}\left(m\right.$-th power subgroup of $\left.H_{q}\right)$ and $H_{p, q}^{\prime}$ ( $p$ and $q$ are relatively prime) have been studied by many authors see [1], [16], [18], [19], [20] and [22]. 
In this paper, we study the commutator subgroups of $H_{p, q}$ and $\bar{H}_{p, q}$. We give the generators, the group structures and the signatures of the commutator subgroups of $H_{p, q}$ and $\bar{H}_{p, q}$. Here we use the Reidemeister-Schreier method, the permutation method (see, [21]) and the extended Riemann-Hurwitz condition (see, [10]) to get our results.

Remark 1. i) The Hecke groups $H_{q}$, the extended Hecke groups $\bar{H}_{q}$ and their normal subgroups have been studied extensively for many aspects in the literature. For examples, see [4], [8], [9], [12] and [14]. Also, there are many relationships between the groups $H_{q}$ (or $\left.\bar{H}_{q}\right)$ and the automorphism groups of Riemann (or Klein) surfaces or of regular maps $\{p, q\}$. Naturally, many results related with $H_{q}$ and $\bar{H}_{q}$ can be generalized to the groups $H_{p, q}$ and $\bar{H}_{p, q}$.

ii) Generalized Hecke groups $H\left(p_{1}, p_{2}, \ldots, p_{n}\right)$ and extended generalized Hecke groups $H^{*}\left(p_{1}, p_{2}, \ldots, p_{n}\right)$ have been introduced first by Huang in [6]. Our studied groups are the special cases $(n=2)$ of these groups given in [6] and [7].

\section{Commutator Subgroups of Generalized Hecke Groups $H_{p, q}$}

In this section, we study the commutator subgroup $H_{p, q}^{\prime}$ of generalized Hecke groups $H_{p, q}$. We use standard notation, in particular, $\left.G^{(} n\right)$ denotes the $n^{t} h$ derived group of a group $G$.

Theorem 1. Let $p$ and $q$ be integers such that $2 \leq p \leq q, p+q>4$.

i) $\left|H_{p, q}: H_{p, q}^{\prime}\right|=p q$.

ii) The commutator subgroup $H_{p, q}^{\prime}$ of $H_{p, q}$ is a free group of rank $(p-1)(q-$

1) with basis $[X, Y],\left[X, Y^{2}\right], \ldots,\left[X, Y^{q-1}\right],\left[X^{2}, Y\right],\left[X^{2}, Y^{2}\right], \ldots,\left[X^{2}, Y^{q-1}\right]$, $\ldots,\left[X^{p-1}, Y\right],\left[X^{p-1}, Y^{2}\right], \ldots,\left[X^{p-1}, Y^{q-1}\right]$. And the signature of $H_{p, q}^{\prime}$ is $\left(\frac{p q-p-q-(p, q)+2}{2} ; \infty^{(p, q)}\right)$ where $(p, q)$ is the greatest common divisor of $p$ and $q$.

iii)For $n \geq 2,\left|H_{p, q}: H_{p, q}^{(n)}\right|=\infty$.

Proof. i) Firstly, we set up the quotient group $H_{p, q} / H_{p, q}^{\prime}$. The quotient group $H_{p, q} / H_{p, q}^{\prime}$ is the group obtained by adding the relation $X Y=Y X$ to the relations of $H_{p, q}$. Thus we have

$$
H_{p, q} / H_{p, q}^{\prime}=<X, Y: X^{p}=Y^{q}=I, X Y=Y X>\simeq C_{p} \times C_{q}
$$

ii) Now we can determine the generators of $H_{p, q}^{\prime}$ by the Reidemeister-Schreier method. To do this, we choose the set $\Sigma=\left\{I, X, X^{2}, \ldots, X^{p-1}, Y, Y^{2}, \ldots\right.$, 
$Y^{q-1}, X Y, X Y^{2}, \ldots, X Y^{q-1}, X^{2} Y, X^{2} Y^{2}, \ldots, X^{2} Y^{q-1}, \ldots, X^{p-1} Y, X^{p-1} Y^{2}, \ldots$ $\left.X^{p-1} Y^{q-1}\right\}$ as a Schreier transversal. All possible products are

$$
\begin{aligned}
& I . X .(X)^{-1}=I, \\
& X . X .\left(X^{2}\right)^{-1}=I, \\
& \vdots \\
& X^{p-1} \cdot X .(I)^{-1}=I,
\end{aligned}
$$

$Y . X .(X Y)^{-1}=Y X Y^{-1} X^{-1}=[Y, X]$,

$Y^{2} \cdot X \cdot\left(X Y^{2}\right)^{-1}=Y^{2} X\left(X Y^{2}\right)^{-1}=\left[Y^{2}, X\right]$

$Y^{q-1}$

$\left.X Y^{q-1}\right)^{-1}=Y^{q-1} X Y^{-(q-1)} X^{-1}=\left[Y^{q-1}, X\right]$

$X Y . X .\left(X^{2} Y\right)^{-1}=X Y X Y^{-1} X^{-2}=[X, Y] \cdot\left[Y, X^{2}\right]$,

$X Y^{2} \cdot X \cdot\left(X^{2} Y^{2}\right)^{-1}=X Y^{2} X Y^{-2} X^{-2}=\left[X, Y^{2}\right] .\left[Y^{2}, X^{2}\right]$,

$$
\begin{aligned}
& \vdots \\
& X Y^{q-1} \cdot X \cdot\left(X^{2} Y^{q-1}\right)^{-1}=X Y^{q-1} X Y^{-(q-1)} X^{-2}=\left[X, Y^{q-1}\right] \cdot\left[Y^{q-1}, X^{2}\right], \\
& X^{2} Y . X .\left(X^{3} Y\right)^{-1}=X^{2} Y X Y^{-1} X^{-3}=\left[X^{2}, Y\right] \cdot\left[Y, X^{3}\right], \\
& X^{2} Y^{2} \cdot X \cdot\left(X^{3} Y^{2}\right)^{-1}=X^{2} Y^{2} X Y^{-2} X^{-3}=\left[X^{2}, Y^{2}\right] \cdot\left[Y^{2}, X^{3}\right], \\
& \quad \vdots \\
& X^{2} Y^{q-1} \cdot X \cdot\left(X^{3} Y^{q-1}\right)^{-1}=X^{2} Y^{q-1} X Y^{-(q-1)} X^{-3}=\left[X^{2}, Y^{q-1}\right] .\left[Y^{q-1}, X^{3}\right], \\
& \quad \vdots \\
& X^{p-1} Y . X .(Y)^{-1}=X^{p-1} Y X Y^{-1}=\left[X^{-1}, Y\right], \\
& X^{p-1} Y^{2} \cdot X .\left(Y^{2}\right)^{-1}=X^{p-1} Y^{2} X Y^{-2}=\left[X^{-1}, Y^{2}\right], \\
& \quad \vdots \\
& X^{p-1} Y^{q-1} \cdot X .\left(Y^{q-1}\right)^{-1}=X^{p-1} Y^{q-1} X Y^{-(q-1)}=\left[X^{-1}, Y^{q-1}\right] .
\end{aligned}
$$

The other products are equal to the identity. Thus, we find the generators of $H_{p, q}^{\prime}$ as $[X, Y],\left[X, Y^{2}\right], \ldots,\left[X, Y^{q-1}\right],\left[X^{2}, Y\right],\left[X^{2}, Y^{2}\right], \ldots,\left[X^{2}, Y^{q-1}\right], \ldots$, $\left[X^{p-1}, Y\right],\left[X^{p-1}, Y^{2}\right], \ldots,\left[X^{p-1}, Y^{q-1}\right]$. The signature of $H_{p, q}^{\prime}$ can be obtained by permutation method and Riemann-Hurtwitz formula.

iii) If we take relations and abelianizing, we find that the resulting quotient is infinite. Thus, it follows that $H_{p, q}^{\prime \prime}$ has infinite index in $H_{p, q}^{\prime}$. Further since this has infinite index it follows that in each group in the derived series from this point on have infinite index.

Example 1. If $p=3$ and $q=4$, then $\left|H_{3,4}: H_{3,4}^{\prime}\right|=12$. We choose $\Sigma=\{I$, $\left.X, X^{2}, Y, Y^{2}, Y^{3}, X Y, X Y^{2}, X Y^{3}, X^{2} Y, X^{2} Y^{2}, X^{2} Y^{3}\right\}$ as a Schreier transversal for $H_{3,4}^{\prime}$. Using the Reidemeister-Schreier method, we get the generators of $H_{3,4}^{\prime}$ as $[X, Y],\left[X, Y^{2}\right],\left[X, Y^{3}\right],\left[X^{2}, Y\right],\left[X^{2}, Y^{2}\right],\left[X^{2}, Y^{3}\right]$. Also the signature of $H_{3,4}^{\prime}$ is $(3 ; \infty)$. 
Corollary 1. If $p=2$, then the generators of $H_{2, q}^{\prime}$ are $[X, Y],\left[X, Y^{2}\right], \ldots$, $\left[X, Y^{q-1}\right]$. Also, the signatures of $H_{2, q}^{\prime}$ is either $\left(\frac{q-1}{2} ; \infty\right)$ if $q$ is odd, or $\left(\frac{q-2}{2} ; \infty^{(2)}\right)$ if $q$ is even. These results coincide with the ones given in [1] and [20], for Hecke groups $H_{q}$.

\section{Commutator Subgroups of Extended Generalized Hecke Groups $\bar{H}_{p, q}$}

In this section, we study the first commutator subgroups $\bar{H}_{p, q}^{\prime}$ of extended generalized Hecke groups $\bar{H}_{p, q}$.

Theorem 2. Let $p$ and $q$ be integers such that $2 \leq p \leq q, p+q>4$.

i) If $p$ and $q$ are both odd numbers, then $\bar{H}_{p, q}^{\prime}=<X, Y: X^{p}=Y^{q}=I>\simeq$ $C_{p} * C_{q}$.

ii) If $p$ is an odd number and $q$ is an even number, then $\bar{H}_{p, q}^{\prime}=<X, Y X Y^{-1}, Y^{2}: X^{p}=\left(Y X Y^{-1}\right)^{p}=\left(Y^{2}\right)^{\frac{q}{2}}=I>\simeq C_{p} * C_{p} * C_{q / 2}$.

iii) If $p$ is an even number and $q$ is an odd number, then $\bar{H}_{p, q}^{\prime}=<X^{2}, Y, X Y X^{-1}:\left(X^{2}\right)^{\frac{p}{2}}=Y^{q}=\left(X Y X^{-1}\right)^{q}=I>\simeq C_{p / 2} * C_{q} * C_{q}$.

$i v)$ If $p$ and $q$ are both even numbers, then $\bar{H}_{p, q}^{\prime}=<X^{2}, Y X^{2} Y^{-1}, Y^{2}$, $X Y^{2} X^{-1}, X Y X Y^{-1}:\left(X^{2}\right)^{p / 2}=\left(Y X^{2} Y^{-1}\right)^{p / 2}=\left(Y^{2}\right)^{q / 2}=\left(X Y^{2} X^{-1}\right)^{q / 2}=$ $I>\simeq C_{p / 2} * C_{p / 2} * C_{q / 2} * C_{q / 2} * \mathbb{Z}$.

Proof. The quotient group $\bar{H}_{p, q} / \bar{H}_{p, q}^{\prime}$ is

$$
\begin{gathered}
\bar{H}_{p, q} / \bar{H}_{p, q}^{\prime}=<X, Y, R: X^{p}=Y^{q}=R^{2}=I, X R=R X^{-1}, Y R=R Y^{-1}, \\
X Y=Y X, X R=R X, Y R=R Y>.
\end{gathered}
$$

i) If $p$ and $q$ are both odd numbers, then from (3.1), we get

$$
\bar{H}_{p, q} / \bar{H}_{p, q}^{\prime}=<R: R^{2}=I>\simeq C_{2} .
$$

Now we can determine the Schreier transversal as $\Sigma=\{I, R\}$. According to the Reidemeister-Schreier method, we can form all possible products :

$$
\begin{array}{lll}
I . X .(I)^{-1}=X, & I . Y .(I)^{-1}=Y, & I . R .(R)^{-1}=I, \\
R . X .(R)^{-1}=R X R, & R . Y .(R)^{-1}=R Y R, & R . R .(I)^{-1}=I .
\end{array}
$$

Since $R X R=X^{-1}$ and $R Y R=Y^{-1}$, the generators of $\bar{H}_{p, q}^{\prime}$ are $X$ and $Y$. Thus we have $\bar{H}_{p, q}^{\prime}=<X, Y: X^{p}=Y^{q}=I>\simeq H_{p, q}$. The signature of $\bar{H}_{p, q}^{\prime}$ is $(0 ; p, q, \infty)$. 
ii) If $p$ is an odd number and $q$ is an even number, then from (3.1), we obtain

$$
\bar{H}_{p, q} / \bar{H}_{p, q}^{\prime}=<Y, R: Y^{2}=R^{2}=I, Y R=R Y>\simeq C_{2} \times C_{2} .
$$

Now we can determine the Schreier transversal as $\Sigma=\{I, Y, R, Y R\}$. According to the Reidemeister-Schreier method, we can form all possible products

$$
\begin{array}{lll}
I . X .(I)^{-1}=X, & I . Y .(Y)^{-1}=I, & I . R .(R)^{-1}=I, \\
Y . X .(Y)^{-1}=Y X Y^{-1}, & Y . Y .(I)^{-1}=Y^{2}, & Y . R .(Y R)^{-1}=I, \\
R . X .(R)^{-1}=R X R, & R . Y .(Y R)^{-1}=R Y R Y^{-1}, & R . R .(I)^{-1}=I, \\
Y R . X .(Y R)^{-1}=Y R X R Y^{-1}, & Y R . Y .(R)^{-1}=Y R Y R, & Y R . R .(Y)^{-1}=I .
\end{array}
$$

After required calculations, we get the generators of $\bar{H}_{p, q}^{\prime}$ as $X, Y X Y^{-1}$ and $Y^{2}$. Thus we obtain $\bar{H}_{p, q}^{\prime}=<X, Y X Y^{-1}, Y^{2}: X^{p}=\left(Y X Y^{-1}\right)^{p}=$ $\left(Y^{2}\right)^{\frac{q}{2}}=I>\simeq C_{p} * C_{p} * C_{q / 2}$. The signature of $\bar{H}_{p, q}^{\prime}$ is $(0 ; p, p,(q / 2), \infty)$.

iii) The proof is similar to ii). Also, the signature of $\bar{H}_{p, q}^{\prime}$ is $(0 ;(p / 2), q, q, \infty)$.

$i v$ ) If $p$ and $q$ are both even numbers, then from (3.1), we find

$$
\begin{aligned}
\bar{H}_{p, q} / \bar{H}_{p, q}^{\prime}=<X, Y, R: X^{2} & =Y^{2}=R^{2}=(X Y)^{2}=(X R)^{2}=(Y R)^{2}=I> \\
& \simeq C_{2} \times C_{2} \times C_{2} .
\end{aligned}
$$

Now we can determine the Schreier transversal as $\Sigma=\{I, X, Y, R, X Y, X R$, $Y R, X Y R\}$. According to the Reidemeister-Schreier method, we can form all possible products :

$$
\begin{array}{ll}
I . X .(X)^{-1}=I, & I . Y .(Y)^{-1}=I, \\
X . X .(I)^{-1}=X^{2}, & X . Y .(X Y)^{-1}=I, \\
Y . X .(X Y)^{-1}=Y X Y^{-1} X^{-1}, & Y . Y .(I)^{-1}=Y^{2}, \\
R . X .(X R)^{-1}=R X R X^{-1}, & R . Y .(Y R)^{-1}=R Y R Y^{-1}, \\
X Y . X .(Y)^{-1}=X Y X Y^{-1}, & X Y . Y .(X)^{-1}=X Y^{2} X^{-1}, \\
X R . X .(R)^{-1}=X R X R, & X R . Y .(X Y R)^{-1}=X R Y R Y^{-1} X^{-1}, \\
Y R . X .(X Y R)^{-1}=Y R X R Y^{-1} X^{-1}, & Y R . Y .(R)^{-1}=Y R Y R, \\
X Y R . X .(Y R)^{-1}=X Y R X R Y^{-1}, & X Y R . Y .(X R)^{-1}=X Y R Y R X^{-1},
\end{array}
$$

The other products are equal to the identity. Since $R X R X^{-1}=X^{-2}$, $X R X R=I, Y R X R Y^{-1} X^{-1}=Y X Y^{-1} X^{-1}, X Y R X R Y^{-1}=X Y X Y^{-1}$, $R Y R Y^{-1}=Y^{-2}, X R Y R Y^{-1} X^{-1}=I, Y R Y R=I$ and $X Y R Y R X^{-1}=$ $X Y^{2} X^{-1}$, we have the generators of $\bar{H}_{p, q}^{\prime}$ as $X^{2}, Y X^{2} Y^{-1}, Y^{2}, X Y^{2} X^{-1}$, $X Y X Y^{-1}$. Then we get $\bar{H}_{p, q}^{\prime}=<X^{2}, Y X^{2} Y^{-1}, Y^{2}, X Y^{2} X^{-1}, X Y X Y^{-1}$ : $\left(X^{2}\right)^{p / 2}=\left(Y X^{2} Y^{-1}\right)^{p / 2}=\left(Y^{2}\right)^{q / 2}=\left(X Y^{2} X^{-1}\right)^{q / 2}=I>\simeq C_{p / 2} * C_{p / 2} *$ $C_{q / 2} * C_{q / 2} * \mathbb{Z}$. Also, the signature of $\bar{H}_{p, q}^{\prime}$ is $\left(0 ;(p / 2)^{(2)},(q / 2)^{(2)}, \infty^{(2)}\right)$. 
Corollary 2. 1) The first commutator subgroup of $\bar{H}_{p, q}$ does not contain any reflection and so $\bar{H}_{p, q}^{\prime}$ is a subgroup of $H_{p, q}$.

2) If $p=2$, then the first commutator subgroups $\bar{H}_{2, q}^{\prime}$ coincide with the ones given in [16] for extended Hecke groups $\bar{H}_{q}$.

3) $H_{p, q}^{\prime} \leq \bar{H}_{p, q}^{\prime} \leq \bar{H}_{p, q}$.

Now we study the second commutator subgroup $\bar{H}_{p, q}^{\prime \prime}$ of $\bar{H}_{p, q}$, except the case $p$ and $q$ are both even numbers, since in this case $\bar{H}_{p, q}^{\prime \prime}$ has infinite index in $\bar{H}_{p, q}$.

Theorem 3. Let $p$ and $q$ be integers such that $2 \leq p \leq q, p+q>4$.

i) If $p$ and $q$ are both odd numbers, then $\bar{H}_{p, q}^{\prime \prime}=H_{p, q}^{\prime}$.

ii) If $p$ is an odd number and $q$ is an even number, then $\bar{H}_{p, q}^{\prime \prime}$ is a free group of rank $(q-1) p^{2}-p q+1$.

iii) If $p$ is an even number and $q$ is an odd number, then $\bar{H}_{p, q}^{\prime \prime}$ is a free group of rank $(p-1) q^{2}-p q+1$.

Proof. The case $i$ ) can be seen from the Theorem 2.1. Since the cases $i i$ ) and iii) are similar, we prove only the case $i$ ii). If we take $a=X^{2}, b=Y$, $c=X Y X^{-1}$, then the quotient group $\bar{H}_{p, q}^{\prime} / \bar{H}_{p, q}^{\prime \prime}$ is the group obtained by adding the relations $a b=b a, a c=c a$ and $b c=c b$ to the relations of $\bar{H}_{p, q}^{\prime}$. Then

$$
\bar{H}_{p, q}^{\prime} / \bar{H}_{p, q}^{\prime \prime} \cong C_{p / 2} \times C_{q} \times C_{q} .
$$

Therefore, we obtain $\left|\bar{H}_{p, q}^{\prime}: \bar{H}_{p, q}^{\prime \prime}\right|=p q^{2} / 2$. Now we choose $\Sigma=\left\{I, a, a^{2}\right.$, $\cdots, a^{(p / 2)-1}, b, b^{2}, \cdots, b^{q-1}, c, c^{2}, \cdots, c^{q-1}, a b, a b^{2}, \cdots, a b^{q-1}, a^{2} b, a^{2} b^{2}, \cdots$, $a^{2} b^{q-1}, \cdots, a^{(p / 2)-1} b, a^{(p / 2)-1} b^{2}, \cdots, a^{(p / 2)-1} b^{q-1}, a c, a c^{2}, \cdots, a c^{q-1}, a^{2} c, a^{2} c^{2}$, $\cdots, a^{2} c^{q-1}, \cdots, a^{(p / 2)-1} c, a^{(p / 2)-1} c^{2}, \cdots, a^{(p / 2)-1} c^{q-1}, b c, b c^{2}, \cdots, b c^{q-1}, b^{2} c$, $b^{2} c^{2}, \cdots, b^{2} c^{q-1}, \cdots, b^{q-1} c, b^{q-1} c^{2}, \cdots, b^{q-1} c^{q-1}, a b c, a b c^{2}, \cdots, a b c^{q-1}, a b^{2} c$, $a b^{2} c^{2}, \cdots, a b^{2} c^{q-1}, \cdots, a b^{q-1} c, a b^{q-1} c^{2}, \cdots, a b^{q-1} c^{q-1}, \cdots, a^{(p / 2)-1} b c, a^{(p / 2)-1} b c^{2}$, $\cdots, a^{(p / 2)-1} b c^{q-1}, a^{(p / 2)-1} b^{2} c, a^{(p / 2)-1} b^{2} c^{2}, \cdots, a^{(p / 2)-1} b^{2} c^{q-1}, \cdots, a^{(p / 2)-1} b^{q-1} c$, $\left.a^{(p / 2)-1} b^{q-1} c^{2}, \cdots, a^{(p / 2)-1} b^{q-1} c^{q-1}\right\}$ as a Schreier transversal for $\bar{H}_{p, q}^{\prime \prime}$.

According to the Reidemeister-Schreier method, we get the generators of $\bar{H}_{p, q}^{\prime \prime}$ as follows. There are total $(p-1) q^{2}-p q+1$ generators obtained by using the theorem of Nielsen in [13]. These generators are $[a, b],\left[a, b^{2}\right], \cdots,\left[a, b^{q-1}\right]$, $\left[a^{2}, b\right],\left[a^{2}, b^{2}\right], \cdots,\left[a^{2}, b^{q-1}\right], \cdots,\left[a^{(p / 2)-1}, b\right],\left[a^{(p / 2)-1}, b^{2}\right], \cdots,\left[a^{(p / 2)-1}, b^{q-1}\right]$, $[a, c],\left[a, c^{2}\right], \cdots,\left[a, c^{q-1}\right],\left[a^{2}, c\right],\left[a^{2}, c^{2}\right], \cdots,\left[a^{2}, c^{q-1}\right], \cdots,\left[a^{(p / 2)-1}, c\right],\left[a^{(p / 2)-1}, c^{2}\right]$, $\cdots,\left[a^{(p / 2)-1}, c^{q-1}\right],[b, c],\left[b, c^{2}\right], \cdots,\left[b, c^{q-1}\right],\left[b^{2}, c\right],\left[b^{2}, c^{2}\right], \cdots,\left[b^{2}, c^{q-1}\right], \cdots$, $\left[b^{q-1}, c\right],\left[b^{q-1}, c^{2}\right], \cdots,\left[b^{q-1}, c^{q-1}\right],[a, b c],\left[a, b^{2} c\right], \cdots,\left[a, b^{q-1} c\right],\left[a, b c^{2}\right],\left[a, b^{2} c^{2}\right]$, $\cdots,\left[a, b^{q-1} c^{2}\right], \cdots,\left[a, b c^{q-1}\right],\left[a, b^{2} c^{q-1}\right], \cdots,\left[a, b^{q-1} c^{q-1}\right],\left[a^{2}, b c\right],\left[a^{2}, b^{2} c\right], \cdots$, 
$\left[a^{2}, b^{q-1} c\right],\left[a^{2}, b c^{2}\right],\left[a^{2}, b^{2} c^{2}\right], \cdots,\left[a^{2}, b^{q-1} c^{2}\right], \cdots,\left[a^{2}, b c^{q-1}\right],\left[a^{2}, b^{2} c^{q-1}\right], \cdots$, $\left[a^{2}, b^{q-1} c^{q-1}\right], \cdots,\left[a^{(p / 2)-1}, b c\right],\left[a^{(p / 2)-1}, b^{2} c\right], \cdots,\left[a^{(p / 2)-1}, b^{q-1} c\right],\left[a^{(p / 2)-1}, b c^{2}\right]$, $\left[a^{(p / 2)-1}, b^{2} c^{2}\right], \cdots,\left[a^{(p / 2)-1}, b^{q-1} c^{2}\right], \cdots,\left[a^{(p / 2)-1}, b c^{q-1}\right],\left[a^{(p / 2)-1}, b^{2} c^{q-1}\right], \cdots$, $\left[a^{(p / 2)-1}, b^{q-1} c^{q-1}\right],[a b, b c],\left[a b, b^{2} c\right], \cdots,\left[a b, b^{q-1} c\right],\left[a b, b c^{2}\right],\left[a b, b^{2} c^{2}\right], \cdots,\left[a b, b^{q-1} c^{2}\right]$, $\cdots,\left[a b, b c^{q-1}\right],\left[a b, b^{2} c^{q-1}\right], \cdots,\left[a b, b^{q-1} c^{q-1}\right],\left[a^{2} b, b c\right],\left[a^{2} b, b^{2} c\right], \cdots,\left[a^{2} b, b^{q-1} c\right]$ $\left[a^{2} b, b c^{2}\right],\left[a^{2} b, b^{2} c^{2}\right], \cdots,\left[a^{2} b, b^{q-1} c^{2}\right], \cdots,\left[a^{2} b, b c^{q-1}\right],\left[a^{2} b, b^{2} c^{q-1}\right], \cdots,\left[a^{2} b, b^{q-1} c^{q-1}\right]$, $\cdots,\left[a^{(p / 2)-1} b, b c\right],\left[a^{(p / 2)-1} b, b^{2} c\right], \cdots,\left[a^{(p / 2)-1} b, b^{q-1} c\right],\left[a^{(p / 2)-1} b, b c^{2}\right],\left[a^{(p / 2)-1} b, b^{2} c^{2}\right]$,

$\cdots,\left[a^{(p / 2)-1} b, b^{q-1} c^{2}\right], \cdots,\left[a^{(p / 2)-1} b, b c^{q-1}\right],\left[a^{(p / 2)-1} b, b^{2} c^{q-1}\right], \cdots,\left[a^{(p / 2)-1} b, b^{q-1} c^{q-1}\right]$.

Also, the signature of $\bar{H}_{p, q}^{\prime \prime}$ is $\left(\frac{q^{2}(p-1)-q(p+1)+2}{2} ; \infty^{(q)}\right)$.

Example 2. Let $p=4$ and $q=5$. Then we have $\left|\bar{H}_{4,5}^{\prime}: \bar{H}_{4,5}^{\prime \prime}\right|=50$. We choose $\Sigma=\left\{I, a, b, b^{2}, b^{3}, b^{4}, c, c^{2}, c^{3}, c^{4}, a b, a b^{2}, a b^{3}, a b^{4}, a c, a c^{2}\right.$, $a c^{3}, a c^{4}, b c, b c^{2}, b c^{3}, b c^{4}, b^{2} c, b^{2} c^{2}, b^{2} c^{3}, b^{2} c^{4}, b^{3} c, b^{3} c^{2}, b^{3} c^{3}, b^{3} c^{4}, b^{4} c$, $b^{4} c^{2}, b^{4} c^{3}, b^{4} c^{4}, a b c, a b c^{2}, a b c^{3}, a b c^{4}, a b^{2} c, a b^{2} c^{2}, a b^{2} c^{3}, a b^{2} c^{4}, a b^{3} c, a b^{3} c^{2}$, $\left.a b^{3} c^{3}, a b^{3} c^{4}, a b^{4} c, a b^{4} c^{2}, a b^{4} c^{3}, a b^{4} c^{4}\right\}$ as a Schreier transversal for $\bar{H}_{4,5}^{\prime \prime}$. According to the Reidemeister-Schreier method, we get total 56 generators of $\bar{H}_{4,5}^{\prime \prime}$ as $[a, b],\left[a, b^{2}\right],\left[a, b^{3}\right],\left[a, b^{4}\right],[a, c],\left[a, c^{2}\right],\left[a, c^{3}\right],\left[a, c^{4}\right],[b, c],\left[b, c^{2}\right]$, $\left[b, b^{3}\right],\left[b, c^{4}\right],\left[b^{2}, c\right],\left[b^{2}, c^{2}\right],\left[b^{2}, c^{3}\right],\left[b^{2}, c^{4}\right],\left[b^{3}, c\right],\left[b^{3}, c^{2}\right],\left[b^{3}, c^{3}\right],\left[b^{3}, c^{4}\right],\left[b^{4}, c\right]$, $\left[b^{4}, c^{2}\right],\left[b^{4}, c^{3}\right],\left[b^{4}, c^{4}\right],[a, b c],\left[a, b^{2} c\right],\left[a, b^{3} c\right],\left[a, b^{4} c\right],\left[a, b c^{2}\right],\left[a, b^{2} c^{2}\right],\left[a, b^{3} c^{2}\right]$, $\left[a, b^{4} c^{2}\right],\left[a, b c^{3}\right],\left[a, b^{2} c^{3}\right],\left[a, b^{3} c^{3}\right],\left[a, b^{4} c^{3}\right],\left[a, b c^{4}\right],\left[a, b^{2} c^{4}\right],\left[a, b^{3} c^{4}\right],\left[a, b^{4} c^{4}\right]$, $[a b, b c],\left[a b, b^{2} c\right],\left[a b, b^{3} c\right],\left[a b, b^{4} c\right],\left[a b, b c^{2}\right],\left[a b, b^{2} c^{2}\right],\left[a b, b^{3} c^{2}\right],\left[a b, b^{4} c^{2}\right],\left[a b, b c^{3}\right]$, $\left[a b, b^{2} c^{3}\right],\left[a b, b^{3} c^{3}\right],\left[a b, b^{4} c^{3}\right],\left[a b, b c^{4}\right],\left[a b, b^{2} c^{4}\right],\left[a b, b^{3} c^{4}\right],\left[a b, b^{4} c^{4}\right]$. Also, the signature of $\bar{H}_{4,5}^{\prime \prime}$ is $(26 ; \infty(5))$.

Corollary 3. Let $p$ and $q$ be integers such that $2 \leq p \leq q, p+q>4$. In case $p$ and $q$ are both even numbers, $\left|\bar{H}_{p, q}: \bar{H}_{p, q}^{(n)}\right|=\infty$ for $n \geq 2$ and, in other cases of $p$ and $q,\left|\bar{H}_{p, q}: \bar{H}_{p, q}^{(n)}\right|=\infty$ for $n \geq 3$.

Corollary 4. If $p=q$ odd number, then the commutator subgroups $\bar{H}_{q, q}^{\prime \prime}$ coincides with the ones given in [15] for extended Hecke groups $\bar{H}_{q}$.

\section{References}

[1] I. N. Cangül and O. Bizim, Commutator subgroups of Hecke groups, Bull. Inst. Math. Acad. Sinica 30 (2002), no. 4, 253-259.

[2] K. Calta and T. A. Schmidt, Infinitely many lattice surfaces with special pseudo-Anosov maps, J. Mod. Dyn. 7, No. 2, 239-254 (2013). 
[3] K. Calta and T. A. Schmidt, Continued fractions for a class of triangle groups, J. Aust. Math. Soc. 93, No. 1-2, 21-42 (2012).

[4] Y.H. He and J. Read, Hecke Groups, Dessins dıEnfants and the Archimedean solids, http://arxiv.org/pdf/1309.2326.

[5] E. Hecke, Über die Bestimmung Dirichletscher Reihen durch ihre Funktionalgleichung, Math. Ann. 112 (1936), 664-699.

[6] S. Huang, Generalized Hecke groups and Hecke polygons, Ann. Acad. Sci. Fenn., Math. 24, No.1, 187-214 (1999).

[7] S. Huang, Realizability of torsion free subgroups with prescribed signatures in Fuchsian groups, Taiwanese J. Math. 13 (2009), no. 2A, 441-457.

[8] S. Ikikardes, R. Sahin and I. N. Cangül, Principal congruence subgroups of the Hecke groups and related results, Bull. Braz. Math. Soc. (N.S.) 40, No. 4, 479-494 (2009).

[9] G. A. Jones, Combinatorial categories and permutation groups, http://arxiv.org/pdf/1309.6119.

[10] R. S. Kulkarni, A new proof and an extension of a theorem of Millington on the modular group, Bull. Lond. Math. Soc. 17, 458-462 (1985).

[11] J. Lehner, Uniqueness of a class of Fuchsian groups, Illinois J. Math. 19 (1975), 308-315.

[12] D. Mayer, T. Mühlenbruch and F. Strömberg, The transfer operator for the Hecke triangle groups, Discrete Contin. Dyn. Syst. 32 (2012), no. 7, 2453-2484.

[13] J. Nielsen, Kommutatorgruppen for det frie produkt af cykliske gruper, Mat. Tidsskr. B (1948), 49-56.

[14] A. D. Pohl, Odd and even Maass cusp forms for Hecke triangle groups, and the billiard flow, http://arxiv.org/pdf/1303.0528.

[15] R. Sahin and O. Bizim, Some subgroups of the extended Hecke groups $\bar{H}\left(\lambda_{q}\right)$, Acta Math. Sci., Ser. B, Engl. Ed. 23, No.4 (2003), 497-502.

[16] R. Sahin, O. Bizim and I. N. Cangül, Commutator subgroups of the extended Hecke groups, Czech. Math. J. 54, No.1 (2004), 253-259.

[17] R. Sahin, S. Ikikardes and Ö. Koruoğlu, Some normal subgroups of the extended Hecke groups $\bar{H}\left(\lambda_{p}\right)$, Rocky Mountain J. Math. 36 (2006), no. 3, 1033-1048. 
[18] R. Sahin and Ö. Koruoğlu, Commutator subgroups of the power subgroups of some Hecke groups, Ramanujan J. 24 (2011), no. 2, 151-159.

[19] R. Sahin and Ö. Koruoğlu, Commutator subgroups of the power subgroups of Hecke groups $H\left(\lambda_{q}\right)$ II, C. R. Math. Acad. Sci. Paris 349 (2011), no. $3-4,127-130$.

[20] T. A. Schmidt and M. Sheingorn, Covering the Hecke triangle surfaces, Ramanujan J. 1 (1997), no. 2, 155-163.

[21] D. Singerman, Subgroups of Fuchsian groups and finite permutation groups, Bull. Lond. Math. Soc. 2, 319-323 (1970).

[22] V. V. Tsanov, Valdemar, Triangle groups, automorphic forms, and torus knots, Enseign. Math. (2) 59 (2013), no. 1-2, 73-113.

Sule KAYMAK (SARICA),

Institute of Science, Department of Mathematics,

Balıkesir University,

Çă̆ış Campus, 10145, Balıkesir, Turkey.

Email: sulekaymak0@gmail.com

Bilal DEMIR,

Necatibey Faculty of Education, Department of Mathematics,

Balıkesir University,

Soma Street, 10100, Balıkesir, Turkey.

Email: bdemir@balikesir.edu.tr

Özden Koruoğlu,

Necatibey Faculty of Education, Department of Mathematics,

Balıkesir University,

Soma Street, 10100, Balıkesir, Turkey.

Email: ozdenk@balikesir.edu.tr

Recep Şahin,

Faculty of Science and Arts, Department of Mathematics,

Balıkesir University,

10145 Çă̆ış Campus, Balıkesir, Turkey

Email: rsahin@balikesir.edu.tr 